\title{
Article \\ Relationship between Swimming Performance, Biomechanical Variables and the Calculated Predicted 1-RM Push-up in Competitive Swimmers
}

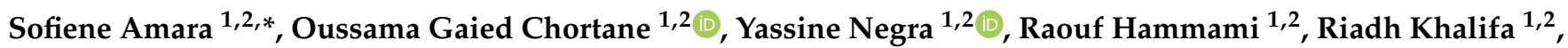 \\ Sabri Gaied Chortane ${ }^{3}$ and Roland van den Tillaar $4, *$ (D)
}

1 Higher Institute of Sport and Physical Education of Ksar-Said, University of La Manouba, Tunis 2010, Tunisia; oussama.gaeid@gmail.com (O.G.C.); yassinenegra@hotmail.fr (Y.N.); raouf.cnmss@gmail.com (R.H.); riadhkhal@yahoo.fr (R.K.)

2 Research Unit (UR17JS01) Sports Performance, Health \& Society, Higher Institute of Sport and Physical Education of Ksar-Said, Universite de la Manouba, Tunis 2010, Tunisia

3 Laboratory of Cardio-Circulatory, Respiratory, Metabolic and Hormonal Adaptations to Muscular Exercise, Faculty of Medicine Ibn El Jazzar, Sousse 4002, Tunisia; Sabrigaied1@gmail.com

4 Department of Sport Sciences and Physical Education, Nord University, 8026 Levanger, Norway

check for

updates

Citation: Amara, S.; Chortane, O.G.; Negra, Y.; Hammami, R.; Khalifa, R.; Chortane, S.G.; van den Tillaar, R Relationship between Swimming Performance, Biomechanical Variables and the Calculated Predicted 1-RM Push-up in Competitive Swimmers. Int. J. Environ. Res. Public Health 2021, 18, 11395. https://doi.org/10.3390/ ijerph182111395

Academic Editors: Antonio José Silva, Daniel Almeida Marinho, Tiago M. Barbosa and Henrique Pereira Neiva

Received: 2 October 2021

Accepted: 27 October 2021

Published: 29 October 2021

Publisher's Note: MDPI stays neutral with regard to jurisdictional claims in published maps and institutional affiliations.

Copyright: (c) 2021 by the authors. Licensee MDPI, Basel, Switzerland. This article is an open access article distributed under the terms and conditions of the Creative Commons Attribution (CC BY) license (https:// creativecommons.org/licenses/by/ $4.0 /)$
* Correspondence: Coachsofieneamara@gmail.com (S.A.); roland.v.tillaar@nord.no (R.v.d.T.)

\begin{abstract}
One repetition maximum (1RM) push-ups, based upon the load-velocity relationship, are able to predict the maximum upper body strength. The aim of the present study was to examine the relationship between the predicted 1RM push-up based upon the load-velocity relationship and swimming performance and kinematical variables in competitive swimmers. Thirty-three competitive male swimmers $($ age $=16.46 \pm 0.59$ years, body mass $=72.82 \pm 8.41 \mathrm{~kg}$, body height $=180.56 \pm 5.69 \mathrm{~cm})$ performed push-up exercises without a weight vest and with a 10, 20 and $30 \mathrm{~kg}$ weight vests. A load-velocity relationship was established as a product of the load and velocity of the push-up per participant, and the equation was used to establish a predicted 1RM. Our findings showed a predicted $1 \mathrm{RM}$ push-up of $82.98 \pm 9.95 \mathrm{~kg}$. Pearson correlations revealed a nearly perfect relationship between the 1RM push-up and the 25 or $50 \mathrm{~m}$ front crawl $(\mathrm{r}=-0.968, \mathrm{r}=-0.955)$, and between 1RM push-up and the 25 or $50 \mathrm{~m}$ front crawl with arms $(\mathrm{r}=-0.955, \mathrm{r}=\mathrm{x} 0.941)$. Similarly, our results revealed significant near-perfect correlations between 1RM push-up and kinematical variables $(r=0.93-0.96)$ except the stroke index, which had a large relationship $(r=0.56)$. This study suggests that swimming performance and kinematical variables are correlated with the predicted 1RM push-up. The 1RM push-up based upon the load-velocity relationship is a low cost and time-effective alternative for swimmers and coaches to predict maximum upper body strength to optimize swimming performance in short races.
\end{abstract}

Keywords: strength; stroke length; stroke rate; upper body

\section{Introduction}

Swimming is a big aquatic sport, with different disciplines, of which the crawl is one. Optimizing front crawl performance requires improving kinematical and physical abilities [1,2]. One of those abilities in front crawl performance is related to the upper body strength $[3,4]$. Specifically, maximum upper and lower body strength exhibit a strong correlation ( $\mathrm{r}=0.67-0.79)$ with swimming performance over less than $100 \mathrm{~m}$ [4].

Therefore, several swimming studies have conducted muscle strength training, aiming to improve the maximum upper and lower body strength in relation to increased swimming performance. Several resistance exercises have been performed, such as the bench press (BP), medicine ball throw (MBT), back squat (BS), and leg extension (LE) with different intensities and loads $[1,5,6]$. In the same context, Lopes et al. [1] revealed that 8 weeks of dry-land training including BP and MBT exercises improved the upper body strength 
(one repetition maximum [1RM] bench press: $12.98 \%$ ), kinematical variables (stroke rate in $50 \mathrm{~m}: 12.20 \%$ and in stroke index in $100 \mathrm{~m}: 5.86 \%)$, and swimming performance $(50 \mathrm{~m}$ : $3.98 \%$ and $100 \mathrm{~m}: 4.34 \%$ ) of competitive sprinter swimmers.

There are different ways to measure upper body strength. For instance, bench press [1,4] and isokinetic dynamometer [5,6] or a Technogym cable crossover apparatus [7] are exercises frequently used as measurements of maximum upper body strength. However, these methods are generally performed in laboratories and weight rooms, and some measurements are very time-consuming or need equipment.

The push-up exercise is a possible exercise that can also measure upper body strength instead of the bench press [8]. Van den Tillaar and Ball [8] showed a nearly perfect correlation between the predicted 1RM push-up and 1RM bench press based upon the load velocity relationship with a submaximal load (ICC $=0.96, r=0.93$ ) in resistance-trained male athletes. While the $1 \mathrm{RM}$ bench press exercise is a good predictor of performance in the 50 and $100 \mathrm{~m}$ front crawl [4] it is not known if this push-up exercise has a good relationship with the kinematical variables and swimming performance.

Therefore, the aim of the present study is to investigate whether the predicted 1RM push-up, based upon the load-velocity relationship of a submaximal load, has a strong relationship with 25 and $50 \mathrm{~m}$ front crawl swimming performance and their kinematical variables (velocity, stroke rate, stroke length, and stroke index) in competitive swimmers. Based upon the study by Keiner et al. [4] that showed a strong correlation between bench press performance and swimming performance, and the study of van den Tillaar and Ball. [8] on similarities between the bench press and push-ups, we hypothesize that there is a very strong correlation between push-ups and swimming performance. If there is a very strong relationship, this could be an easier and cheaper method to help coaches and swimmers evaluate upper body strength in relation to swimming performance.

\section{Materials and Methods}

\subsection{Experimental Approach to the Problem}

To investigate the relationship between maximum upper body strength determined by the predicted 1-RM push-up and swimming performance variables, a load-velocity relationship was established by evaluating the velocity at four different sub-maximum loads in push-ups. The 1RM push-up was predicted based on the load-velocity relationship established by a prediction equation. The predicted 1-RM push-up load was used as the independent variable, while the swimming performance ( 25 and $50 \mathrm{~m}$ front crawl, and 25 and $50 \mathrm{~m}$ front crawl with arms only) and kinematical variables of the $50 \mathrm{~m}$ front crawl (velocity, stroke length, stroke rate, and stroke index) were the dependent variables. The present study was performed during the competitive season (February-April).

\subsection{Participants}

Thirty-three national competitive male swimmers (age $=16.46 \pm 0.59$ years, body mass $=72.82 \pm 8.41 \mathrm{~kg}$, body height $=180.56 \pm 5.69 \mathrm{~cm}$ ) with previous dry land resistance training experience ( $6.08 \pm 0.37$ years) and swimming training experience (9.50 \pm 0.71 years) volunteered to participate in the study. Swimmers performed approximately six watertraining sessions per week (between 4000 and $6000 \mathrm{~m}$ per session) and two dryland sessions (general strength training) before the present study. The swimmers were sprinters (mean personal best performance in $50 \mathrm{~m}$ front crawl was $25.01 \mathrm{~s}$ ). Participants were asked to avoid any additional resistance training targeting the upper body for $72 \mathrm{~h}$ before the test. Written consent was obtained from each swimmer and their guardians prior to the study. This investigation complied with the ethical regulations in force for research and was approved by the ethics committee of Higher Institute of Sport and Physical Education of Ksar Said, University of Manouba, Tunisia (Research Unit UR17JS01, Sports Performance, Health and Society) and in line with the underlying the latest Declaration of Helsinki of 2013. All physical experience and anthropometric characteristics are presented in Table 1. 
Table 1. Physical experience and anthropometric characteristics of the swimmers (mean $\pm \mathrm{SD}$ ).

\begin{tabular}{cc}
\hline Swimmers Characteristics & National Competitive Male Swimmers (N = 33) \\
\hline Age (yr-old) & $16.46 \pm 0.59$ \\
Height (cm) & $180.56 \pm 5.69$ \\
Body mass (kg) & $72.82 \pm 8.41$ \\
Swimming training experience (years) & $9.50 \pm 0.71$ \\
Resistance training experience (years) & $6.08 \pm 0.37$ \\
\hline
\end{tabular}

\subsection{Procedures}

\subsubsection{Maximum Strength Prediction Test}

The swimmers visited the laboratory $\left(22.3{ }^{\circ} \mathrm{C}\right.$ air temperature, $45 \%$ relative humidity) twice, separated by $48 \mathrm{~h}$, at the same time, on two days (10.00 AM) and all participants were informed to eat their breakfast minimum $3 \mathrm{~h}$ before the start of the test, and to avoid all other nutrition, including caffeine or other nutrition. The first time was for a familiarization session. The second visit was for a test session, which started with the anthropometric measurements of the swimmers (height and body mass). Subsequently, each subject performed a standardized warm-up consisting of a $5 \mathrm{~min}$ run on a treadmill at a submaximal level $(8-10 \mathrm{~km} / \mathrm{h})$. After the warm-up, participants performed the push-up test with four different loads: own body mass and with a 10, 20, and $30 \mathrm{~kg}$ weight vests (Titan Fitness, Memphis, TN, USA). Three repetitions per load were performed, and the different loads were given in ascending or descending order, equally randomized for each subject [8]. Rest intervals of 3 to $5 \mathrm{~min}$ were allowed between each load to avoid fatigue. Participants used their preferred grip width in the push-up, and this was measured and normalized for each load maintained during the exercise. The push-up depth was qualitatively validated by the main height of each repetition. To avoid risk of injury, no actual 1RM testing was performed in this study. The push handles were placed on a force plate (9290AD; Kistler, Winterthur, Switzerland), which sampled at $500 \mathrm{~Hz}$. The participant's feet were placed behind the plate at the same height as the force plate (Figure 1). The initial ground reaction force was measured when the participant was in the starting position and bearing their full weight on the lift handles. The reaction force of the ground at the starting position was then used to calculate the absolute load and the percentage of body weight (without and with weight vest) to be lifted during the different push-up conditions [8]. The test was controlled by two strength and conditioning coaches.

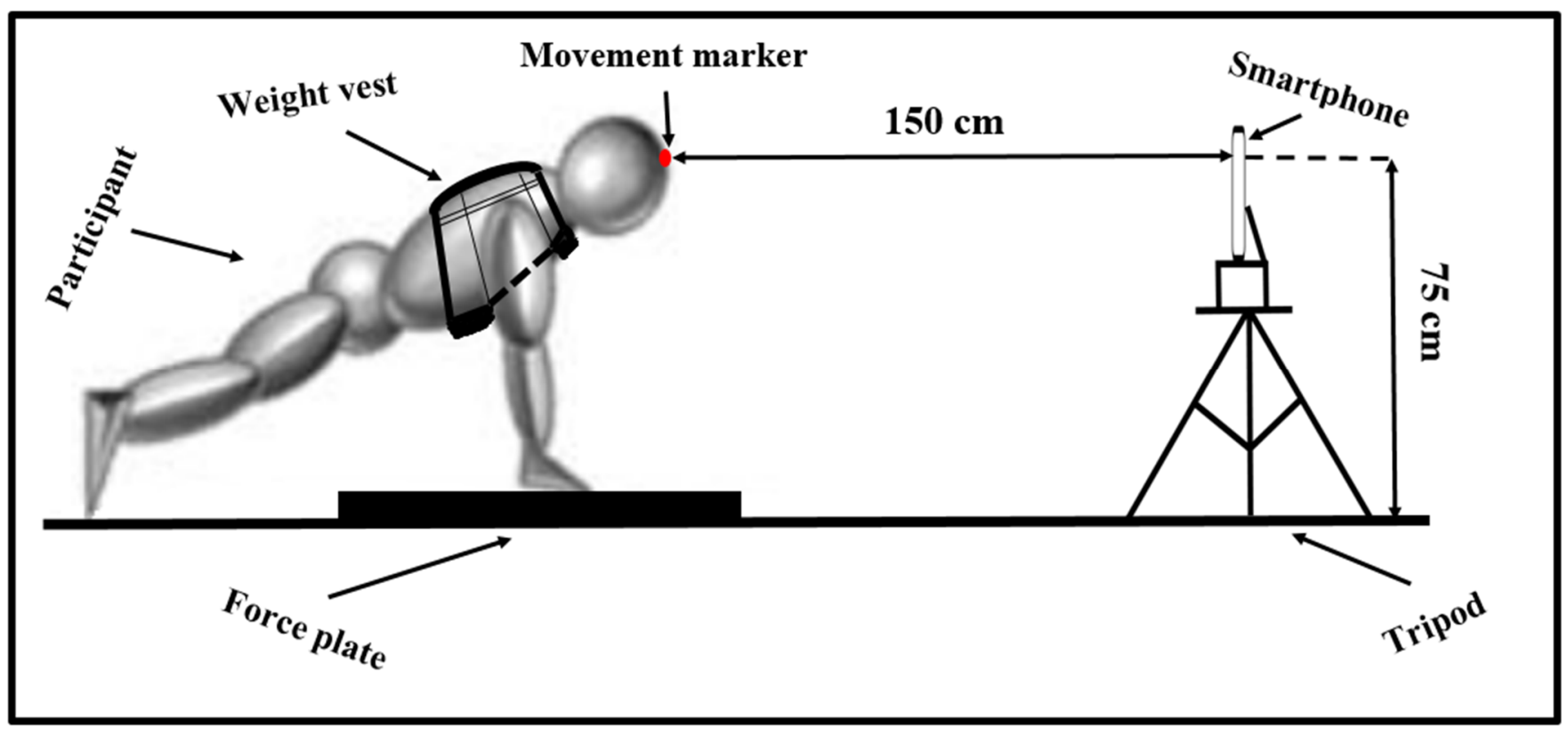

Figure 1. Push-up test condition performed by the participant and the setting up of test equipment. 
A smartphone (Redmi Note 8, Xiaomi, Beijing, China) with a high-speed camera (Snapdragon 886 processor at $2.0 \mathrm{GHz}$ and $48+8+2$-megapixel quad-cameras) was used to film the push-up exercises. All videos were recorded in the slow-motion option at $240 \mathrm{fps}$ in automatic mode. The smartphone was attached to a tripod $150 \mathrm{~cm}$ from the focal plane to the plane of the movement marker placed on the participant's head. In addition, the main lens of the camera was placed at a $75 \mathrm{~cm}$ height [9]. The video recordings of the push-ups were processed with Kinovea software, version 0.8.15 (Joan Charmant \& Contrib., kinovea.org, accessed on 25 October 2021) [10] with a frequency of $240 \mathrm{~Hz}$ to ensure the correct calculation of the execution speed [11-13]. A point marker was put on the head of each swimmer. In addition, the displacement of the point maker during the descending and ascending phases of the push-up was limited by two imaginary lines added to the 2D plane in the Kinovea software. The mean concentric velocity was calculated from the distance between the lowest position and the highest position according to the imaginary digital lines, and time it took to cover this distance.

The load-velocity relationship was established for the push-up exercise by the average velocity of the three repetitions of the four different loads performed by each participant, as determined by the Kinovea software. Based on the athlete's performance at different loads, linear regression was used to predict the 1RM push-up for each participant.

To calculate the predicted 1RM push-up, the following formula was used:

$$
y=a \times x+b
$$

The variable $x$ was set at $0.18 \mathrm{~m} / \mathrm{s}$, which indicated the minimum mean propulsive velocity at which $1 \mathrm{RM}$ was theoretically achievable [14]. Additionally, the coefficient of $x$ (a) and y-intercept (b) were individualized for each participant. To establish a and b in the linear equation for each participant, scatter plots were produced, and a linear regression line was added using Microsoft Excel (version 18.12, Microsoft, Redmond, Washington, USA).

\subsubsection{Swimming Performances Tests}

After a standard $800 \mathrm{~m}$ warm-up (600 $\mathrm{m}$ aerobic swim $+200 \mathrm{~m}$ progressive sprint), the 25 and $50 \mathrm{~m}$ front crawl tests were performed with a diving start. Five minutes of rest were integrated between the two tests. Thirty minutes after the $50 \mathrm{~m}$ front crawl test, the 25 and $50 \mathrm{~m}$ front crawl tests with arms only were performed with a water start. A pull buoy and an elastic between the two ankles were used to eliminate the effect of the legs during swimming. Five minutes of rest were performed between the two tests. All swimming performances were timed in seconds, as recorded by two timekeeping specialists with a stopwatches (SEIKO S120-4030, Tokyo, Japan) and were performed in a $50 \mathrm{~m}$ indoor pool with 27.2 and $25.9{ }^{\circ} \mathrm{C}$ water and air temperatures, respectively, and $64 \%$ relative humidity at $10.00 \mathrm{AM}$. All participants were informed, as in the dry land test, to eat their breakfast $3 \mathrm{~h}$ before the start of the test, and to avoid all other nutrition, including caffeine.

\subsubsection{Biomechanical Variable Tests}

To eliminate the start and end effects of the race, only a distance of $10 \mathrm{~m}$ (between the 7.5 and $17.5 \mathrm{~m}$ marks) was evaluated for the kinematical variables of the $50 \mathrm{~m}$ front crawl. A surface video camera, Sony SNC VB 603 ( $50 \mathrm{~Hz}$, full HD, 1080 p), was used to record the kinematical variables. The camera was placed about $5 \mathrm{~m}$ above the water and about $10 \mathrm{~m}$ away from the swimming line, laterally to the $10 \mathrm{~m}$ measuring zone. In addition, Kinovea software version 0.8.15 (Joan Charmant \& Contrib., kinovea.org) was used to analyze the video sequences. The velocity was determined from the time taken to cover the $10 \mathrm{~m}$. The stroke rate (SR) was assessed from the time taken to complete three consecutive stroke cycles. In addition, the stroke length (SL) was calculated from the ratio between the velocity and the corresponding stroke rate [15]. The stroke index (SI) was computed by multiplying velocity by stroke length $[16,17]$. 


\subsection{Statistical Analyses}

SPSS 26.0 (SPSS Inc., Chicago, IL, USA) was used for statistical analysis. All data are presented as mean and standard deviation. Normality was assessed for all variables using the Kolmogorov-Smirnov test, and all variables were normally distributed $(p>0.05)$. Linear regression analysis was used to predict the 1RM push-up performance. In addition, relationships between the $1 \mathrm{RM}$ push-up and swimming performance were determined by the Pearson product-moment correlation. Therefore, the correlation coefficients were interpreted as: small ( 0.1 to 0.3$)$, moderate $(0.3$ to 0.5$)$, large ( 0.5 to 0.7$)$, very large ( 0.7 to 0.9 ), and nearly perfect (0.9 to 1.0$)$ [18].

\section{Results}

Our results showed that the predicted $1 \mathrm{RM}$ push-up was $82.98 \pm 9.95 \mathrm{~kg}$. However, the load lifted during push-up conditions was between $44.69 \pm 7.55 \mathrm{~kg}$ for the own body mass condition and $66.67 \pm 8.66 \mathrm{~kg}$ for the $+30 \mathrm{~kg}$ condition. For instance, the percentage of body mass (+ weight vest) that had to be lifted during the push-up conditions was from $61.0 \pm 3.47 \%$ to $64.6 \pm 3.1 \%$. The mean velocities during the lifts with different loads in the push-up were from $0.85 \pm 0.07 \mathrm{~m} \cdot \mathrm{s}^{-1}$ for the own body mass condition to $0.46 \pm 0.07 \mathrm{~m} \cdot \mathrm{s}^{-1}$ for the $+30 \mathrm{~kg}$ condition. A significant nearly perfect correlation was observed between the mean velocity at each load for each participant and the percentage of 1RM in the push-up $(r=-0.94$, Table 2 and Figure 2).

Table 2. Mean load and velocity in the four push-up conditions.

\begin{tabular}{ccccc}
\hline Load & $\mathbf{1}$ & $\mathbf{2}$ & $\mathbf{3}$ & $\mathbf{4}$ \\
\hline Push-up conditions & $0 \mathrm{~kg}$ & $+10 \mathrm{~kg}$ & $+20 \mathrm{~kg}$ & $+30 \mathrm{~kg}$ \\
Body mass plus weight vest $(\mathrm{kg})$ & $72.82 \pm 8.41$ & $82.82 \pm 8.41$ & $92.82 \pm 8.41$ & $102.82 \pm 8.41$ \\
Actual load lifted $(\mathrm{kg})$ & $44.69 \pm 7.55$ & $52.20 \pm 8.12$ & $59.11 \pm 8.31$ & $66.67 \pm 8.66$ \\
\% of body mass (+weight vest) & $61.00 \pm 3.37$ & $62.69 \pm 3.49$ & $63.41 \pm 3.18$ & $64.60 \pm 3.14$ \\
Mean velocity $\left({\mathrm{m} \cdot \mathrm{s}^{-1} \text { ) }}^{0.85 \pm 0.07}\right.$ & $0.76 \pm 0.07$ & $0.65 \pm 0.06$ & $0.46 \pm 0.07$ \\
\hline
\end{tabular}

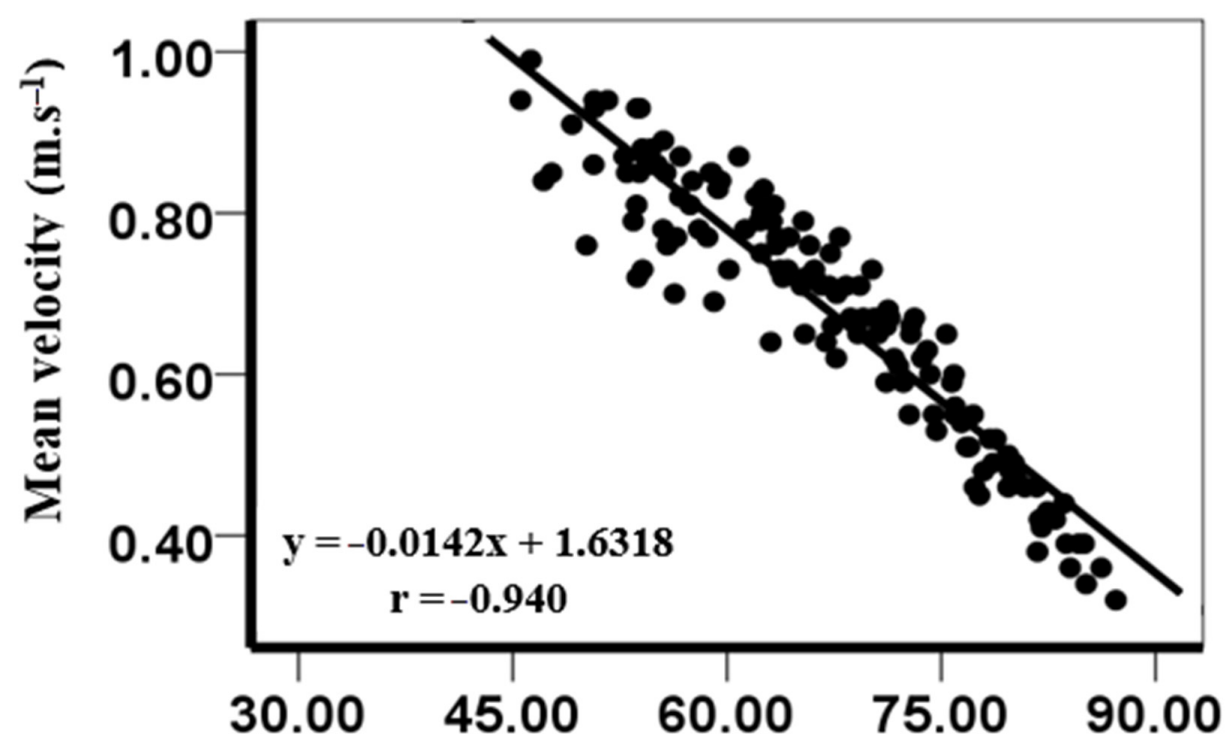

\section{Percentage of the predicted 1RM push-up (kg)}

Figure 2. Relationship between mean velocity at each load and the percentage of the 1RM push-up.

The results of the present study revealed a significant nearly perfect correlation between the calculated predicted 1RM push-up and the $25(\mathrm{r}=-0.96)$ and $50 \mathrm{~m}$ front crawl $(r=-0.97)$. Our results also indicated a significant nearly perfect correlation between 
the 1RM push-up and $25(\mathrm{r}=-0.94)$ and $50 \mathrm{~m}(\mathrm{r}=-0.96)$ front crawl with arms only (Figure 3).

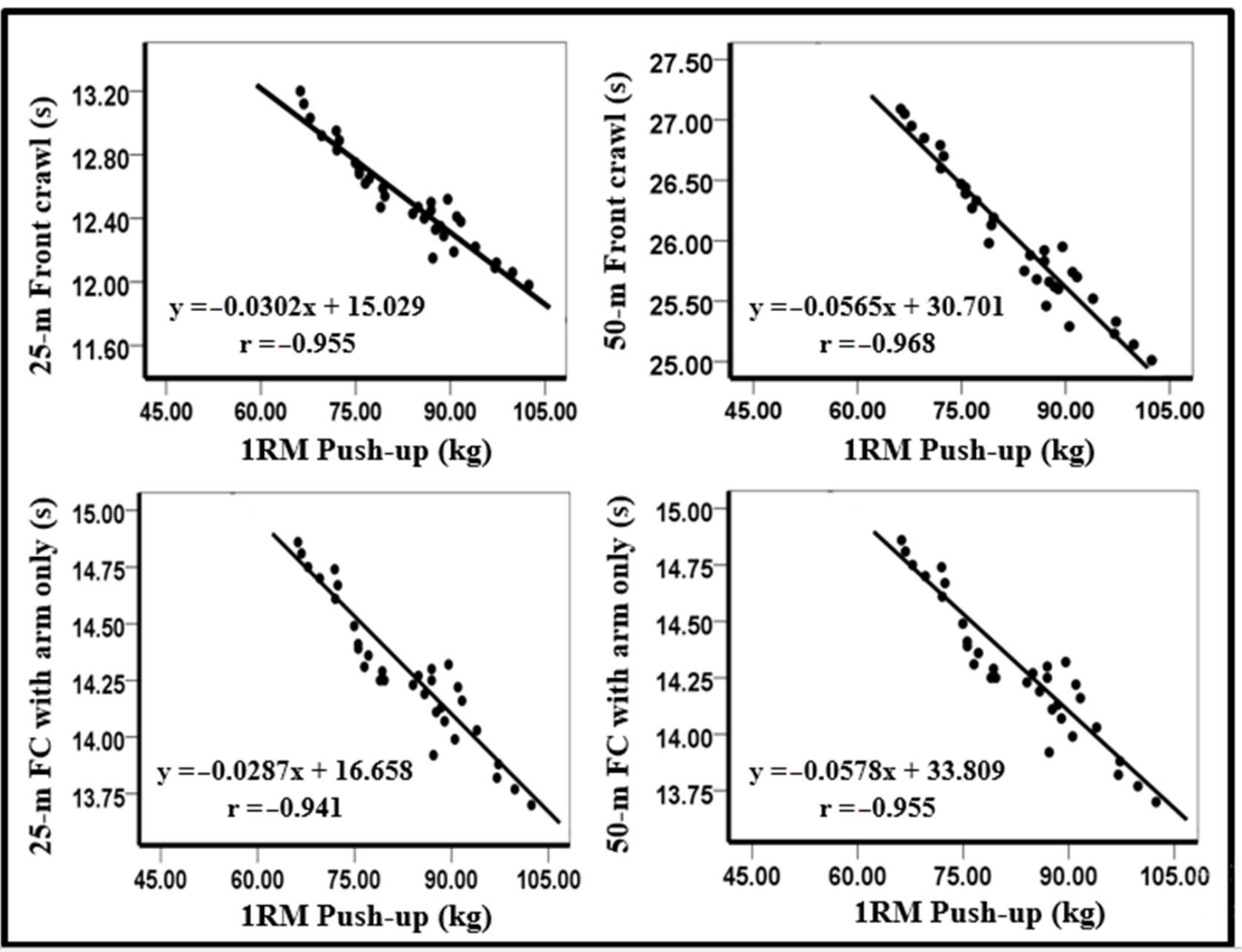

Figure 3. Relationship between swimming performance and the calculated predicted 1-RM push-up. FC: front crawl.

Furthermore, significant nearly perfect correlations were observed between the 1RM push-up and the kinematical variables velocity $(r=0.96)$, stroke length $(r=-0.93)$, and stroke rate $(\mathrm{r}=0.96)$ in the $50 \mathrm{~m}$ front crawl, while a large significant relationship was found between the 1RM push-up and stroke index $(r=0.562$, Figure 4$)$. 

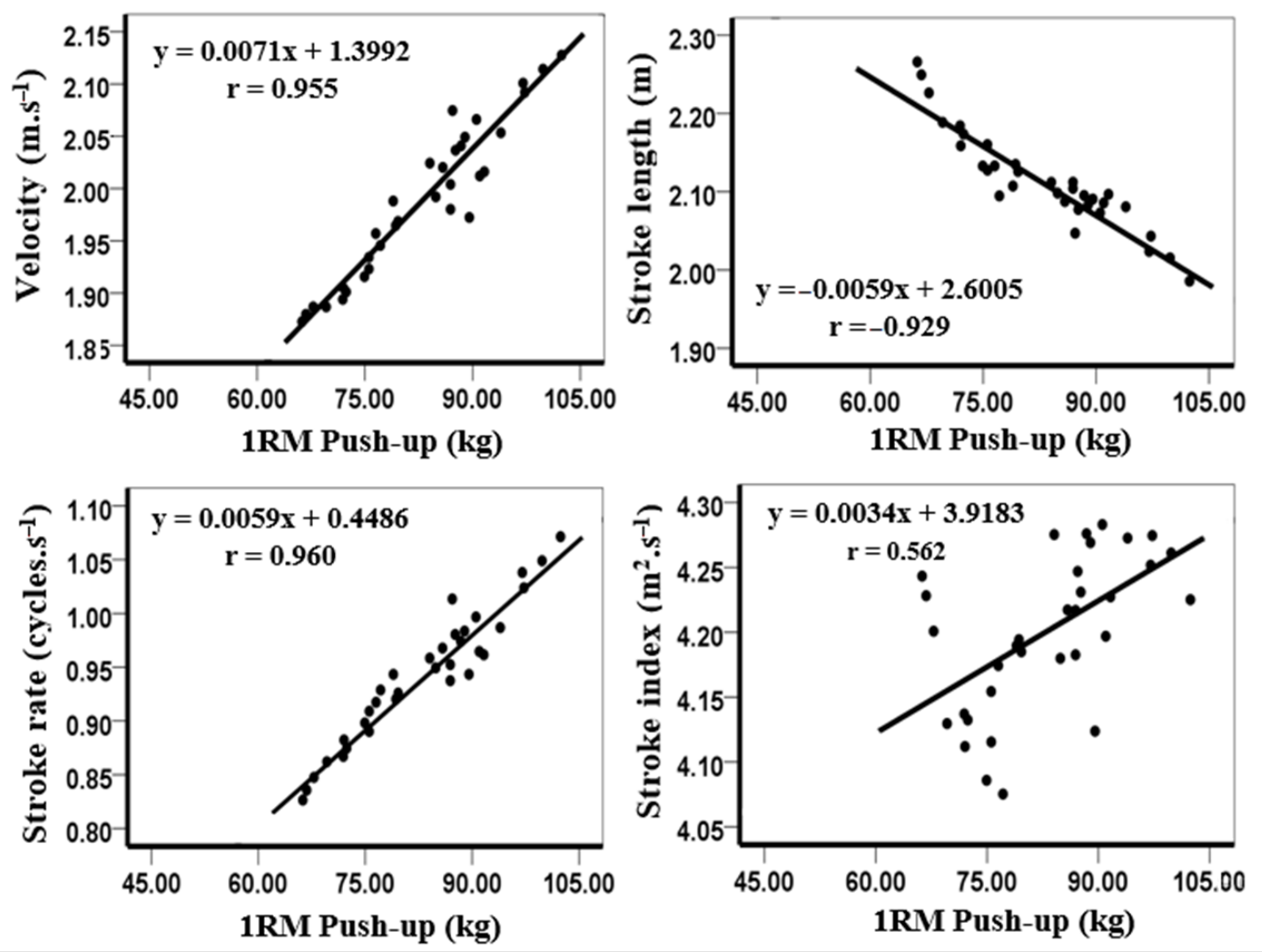

Figure 4. Relationship between kinematical variables and the calculated predicted 1-RM push-up.

\section{Discussion}

This study aimed to investigate the correlation between the calculated predicted $1 \mathrm{RM}$ push-up based upon the load-velocity relationship, swimming performance, and kinematical variables in the $50 \mathrm{~m}$ front crawl in competitive swimmers.

Our results showed a nearly perfect association between the 1RM push-up, all front crawl swimming performances and most kinematical variables during the $50 \mathrm{~m}$ crawl. Since no study has investigated the relationship between push-up performance and swimming performance, it is difficult to compare the findings with other studies. However, Keiner et al. [4] studied the correlation between the maximum upper and lower body strength and swimming performance (50 and $100 \mathrm{~m}$ front crawl) and found large to very large correlations with the $1 \mathrm{RM}$ bench press and back squat $(\mathrm{r}=0.79, \mathrm{r}=0.67$, respectively) in moderately trained male swimmers (age $=17.5 \pm 1.6$ years). In young male water polo players (age $=11.9 \pm 1.3$ years), Keiner et al. [3] showed a significant correlation between the 15 and $25 \mathrm{~m}$ front crawl and the $1 \mathrm{RM}$ bench press and arm span $(\mathrm{r}=0.71$, $r=0.72$, respectively). As van den Tillaar and Ball. [8] showed that there is a near-perfect correlation between maximal bench press performance and push-ups, it is possible to compare these previous studies using the bench press with the present study. Therefore, testing upper body strength measured with push-ups seems to be a good predictor for front crawl swimming performance in competitive swimmers.

Furthermore, our results also showed a nearly perfect correlation between the 1RM push-up and 25 and $50 \mathrm{~m}$ swimming performance with arms only. For, instance Morouço et al. [19] showed that swimming with arms only accounts for $70.3 \%$ of the estimated 
relative contribution in the $50 \mathrm{~m}$ front crawl. The same authors [19] showed that to improve the $50 \mathrm{~m}$ velocity, swimmers are highly dependent on the maximum forces they can perform with arms only $(r=0.77)$. For this, it is very important to increase the maximum upper body strength to improve force propulsion in the front crawl with arms only and, consequently, to optimize the swimming performance [19].

Moreover, the predicted 1RM push-up showed very large to nearly perfect relationships with the kinematical variables, which was not surprising. However, the velocity was also calculated by multiplying the stroke rate by the stroke length $(\mathrm{V}=\mathrm{SR} \times \mathrm{SL})$. In addition, an increase in SR and/or SL increased the velocity [20]. However, short swimming distances are characterized by high velocity and maximum upper body strength $[4,20,21]$. Earlier studies only investigated the kinematical variables in relation to muscle strength by using the torque measured during isometric and isokinetic measurements of the upper body. Gola et al. [22] also found a significant correlation between the 25 and $50 \mathrm{~m}$ swimming velocity in the front crawl and the relative sum of the total torque for the upper extremity muscles $(r=0.60, r=0.57$, respectively) in university students with 2 years of mean competitive swimming experience. However, the difference in the years of experience in resistance training among the university students [22] and the competitive swimmers participating in this study may explain the discrepancy in the results obtained with this earlier study.

That such a high correlation was found between the maximal push-up performance and front crawl swimming performance over short distances and its kinematical variables is not surprising, since most of the muscles used during the push-up, i.e.: the shoulder muscles (rotator cuff, trapezius, deltoid) and arm muscles (biceps brachii and triceps brachii), are among the main muscles active during the front crawl [23].

During the push-up, around 61 to $65 \%$ of body mass (no extra load-30 kg weight vest respectively) were the loads lifted, which is in accordance with the findings of van den Tillaar and Ball. [8]. This is important information when planning to evaluate push-up performance based upon the load-velocity relationship when you have no access to a force plate to measure the forces. Furthermore, this information is also useful when using push-ups with extra loads (e.g., a weight vest) in planning training.

On the other hand, maximum upper body strength and swimming performance were improved by training programs that included the bench press (BP) and medicine ball throw (MBT) exercises [1,15]. Amara et al. [15] showed that nine weeks of dry land training with BP and MBT can improve the performance of 1 RM BP $(12.11 \pm 1.79 \%)$ and sprint swimming performances of the $50 \mathrm{~m}$ front crawl $(4.22 \pm 0.18 \%)$ in male competitive swimmers (age $=16.5 \pm 0.30$ years). In the same context, Lopes et al. [1] revealed that eight weeks of dry-land training including BP and MBT can improve the 1RM BP $(14.92 \%)$ and the performance of $50 \mathrm{~m}$ front crawl (3.98\%) in university swimmers of the national level (age $=20.55 \pm 1.76$ years). According to our results, nearly perfect relationships were found between the 1RM push-up and swimming performance. For this reason, push-up exercises can be also included in the future studies that verify the effect of dry land training on swimming performance.

This study has some methodological limitations that warrant discussion. First, the participants may not have much experience with push-ups with weighted vests of different loads, which could influence the load-velocity relationship and, therefore, the predicted 1RM. In addition, the actual 1RM push-up was not been established, so it was not possible to make a comparison of the predicted 1RM to the actual 1RM. In a future study, an upper body training intervention is suggested, in which push-up performance is tested to investigate if swimming performance increases as much as push-up performance. In addition, in future studies it is necessary to examine the relationship between 1RM pushup prediction and swimming performance in female swimmers, and in swimmers of other training levels and other age categories (e.g., pre-pubertal or senior swimmers). On the other hand, the testing of swimming performance using a stopwatch represents a 
limitation in terms of test accuracy. Future studies should use electronic timing systems (e.g., Omega system).

\section{Conclusions}

The outcomes of this study revealed nearly perfect relationships between the 1RM push-up, swimming performance, and kinematical variables (velocity, stroke rate, stroke length, and stroke index) in the front crawl. This method is a low cost and time-effective alternative. For this reason, strength and conditioning coaches and swimmers could utilize the push-up exercise to predict maximum upper body strength to optimize swimming performance in short races.

Author Contributions: Conceptualization, S.A. and R.v.d.T.; formal analysis, S.A. and R.v.d.T.; methodology, S.A. and S.G.C.; writing-original draft, Y.N. and R.H. and writing-review and editing, O.G.C., S.A., Y.N., R.v.d.T. and R.K. All authors have read and agreed to the published version of the manuscript.

Funding: This research received no external funding.

Institutional Review Board Statement: The study was conducted according to the guidelines of the Declaration of Helsinki and approved by the ethical advisory committee of the Research Unit (UR17JS01) Sports Performance, Health and Society, Higher Institute of Sport and Physical Education of Ksar Saîd, Universite de la Manouba, Tunis, 2010, Tunisia.

Informed Consent Statement: Informed consent was obtained from all subjects and guardians involved in the study.

Data Availability Statement: The data presented in this study are available on reasonable re-quest from the corresponding author.

Acknowledgments: The authors thank all the subjects who participated in this study, and all individuals included in this section have consented to the acknowledgement.

Conflicts of Interest: The authors declare no conflict of interest.

\section{References}

1. Lopes, T.J.; Neiva, H.P.; Gonçalves, C.A.; Nunes, C.; Marinho, D.A. The effects of dry-land strength training on competitive sprinter swimmers. J. Exerc. Sci. Fit. 2021, 19, 32-39. [CrossRef]

2. Sammoud, S.; Negra, Y.; Bouguezzi, R.; Hachana, Y.; Granacher, U.; Chaabene, H. The effects of plyometric jump training on jump and sport-specific performances in prepubertal female swimmers. J. Exerc. Sci. Fit. 2021, 19, 25-31. [CrossRef]

3. Keiner, M.; Rähse, H.; Wirth, K.; Hartmann, H.; Fries, K.; Haff, G.G. Influence of Maximal Strength on In-Water and Dry-Land Performance in Young Water Polo Players. J. Strength Cond. Res. 2020, 34, 1999-2005. [CrossRef] [PubMed]

4. Keiner, M.; Wirth, K.; Fuhrmann, S.; Kunz, M.; Hartmann, H.; Haff, G.G. The influence of upper- and lower-body maximum strength on swim block start, turn, and overall swim performance in sprint swimming [published online ahead of print, 2019 Aug 15]. J. Strength Cond. Res. 2019, 10.1519/JSC. [CrossRef]

5. Girold, S.; Jalab, C.; Bernard, O.; Carette, P.; Kemoun, G.; Dugué, B. Dry-land strength training vs. electrical stimulation in sprint swimming performance. J. Strength Cond. Res. 2012, 26, 497-505. [CrossRef]

6. Girold, S.; Maurin, D.; Dugué, B.; Chatard, J.C.; Millet, G. Effects of dry-land vs. resisted- and assisted-sprint exercises on swimming sprint performances. J. Strength Cond. Res. 2007, 21, 599-605. [CrossRef]

7. Aspenes, S.; Kjendlie, P.L.; Hoff, J.; Helgerud, J. Combined strength and endurance training in competitive swimmers. J. Sports Sci. Med. 2009, 8, 357-365. [PubMed]

8. Van den Tillaar, R.; Ball, N. Push-Ups are Able to Predict the Bench Press 1-RM and Constitute an Alternative for Measuring Maximum Upper Body Strength Based on Load-Velocity Relationships. J. Hum. Kinet. 2020, 73, 7-18. [CrossRef]

9. Jimenez-Olmedo, J.M.; Penichet-Tomás, A.; Villalón-Gasch, L.; Pueo, B. Validity and reliability of smartphone high-speed camera and Kinovea for velocity-based training measurement. J. Hum. Sport Exerc. 2021, 16. [CrossRef]

10. Puig-Diví, A.; Escalona-Marfil, C.; Padullés-Riu, J.M.; Busquets, A.; Padullés-Chando, X.; Marcos-Ruiz, D. Validity and reliability of the Kinovea program in obtaining angles and distances using coordinates in 4 perspectives. PLOS ONE. 2019, 14, e0216448. [CrossRef]

11. Velázquez, J.S.; Iznaga-Benítez, A.M.; Robau-Porrúa, A.; Sáez-Gutiérrez, F.L.; Cavas, F. New Affordable Method for Measuring Angular Variations Caused by High Heels on the Sagittal Plane of Feet Joints during Gait. Appl. Sci. 2021, 11, 5605. [CrossRef]

12. Muro-De-La-Herran, A.; Garcia-Zapirain, B.; Mendez-Zorrilla, A. Gait analysis methods: An overview of wearable and nonwearable systems, highlighting clinical applications. Sensors 2014, 14, 3362-3394. [CrossRef] 
13. Wang, M.; Gu, Y.; Baker, J.S. Analysis of foot kinematics wearing high heels using the Oxford foot model. Technol. Health Care 2018, 26, 815-823. [CrossRef] [PubMed]

14. González-Badillo, J.J.; Marques, M.C.; Sánchez-Medina, L. The importance of movement velocity as a measure to control resistance training intensity. J. Hum. Kinet 2011, 29A, 15-19. [CrossRef]

15. Amara, S.; Barbosa, T.M.; Negra, Y.; Hammami, R.; Khalifa, R.; Chortane, S.G. The Effect of Concurrent Resistance Training on Upper Body Strength, Sprint Swimming Performance and Kinematics in Competitive Adolescent Swimmers. A Randomized Controlled Trial. Int. J. Environ. Res. Public Health 2021, 18, 10261. [CrossRef]

16. Costill, D.L.; Kovaleski, J.; Porter, D.; Kirwan, J.; Fielding, R.; King, D. Energy expenditure during front crawl swimming: Predicting success in middle-distance events. Int. J. Sports. Med. 1985, 6, 266-270. [CrossRef] [PubMed]

17. Zacca, R.; Azevedo, R.; Peterson Silveira, R.; Vilas-Boas, J.P.; Pyne, D.B.; Castro, F.A.S.; Fernandes, R.J. Comparison of Incremental Intermittent and Time Trial Testing in Age-Group Swimmers. J. Strength Cond. Res. 2019, 33, 801-810. [CrossRef]

18. Hopkins, W.G.; Marshall, S.W.; Batterham, A.M.; Hanin, J. Progressive statistics for studies in sports medicine and exercise science. Med. Sci. Sports. Exerc. 2009, 41, 3-13. [CrossRef]

19. Morouço, P.G.; Marinho, D.A.; Izquierdo, M.; Neiva, H.; Marques, M.C. Relative Contribution of Arms and Legs in 30 s Fully Tethered Front Crawl Swimming. Biomed. Res. Int. 2015, 2015, 563206. [CrossRef] [PubMed]

20. Craig, A.B., Jr.; Skehan, P.L.; Pawelczyk, J.A.; Boomer, W.L. Velocity, stroke rate, and distance per stroke during elite swimming competition. Med. Sci. Sports. Exerc. 1985, 17, 625-634. [CrossRef]

21. Strass, D. Effects of maximal strength training on sprint performance of competitive swimmers. Swim. Sci. V 1988, 16, 149-156.

22. Gola, R.; Urbanik, C.; Iwańska, D.; Madej, A. Relationship between muscle strength and front crawl swimming velocity. Hum. Mov. 2014, 15, 110-115. [CrossRef]

23. Martens, J.; Figueiredo, P.; Daly, D. Electromyography in the four competitive swimming strokes: A systematic review. J. Electromyogr. Kinesiol. 2015, 25, 273-291. [CrossRef] [PubMed] 